\title{
ELABORACIÓN DE UN CUESTIONARIO DIRIGIDO AL PROFESORADO UNIVERSITARIO ANTE EL ÁREA EUROPEA DE EDUCACIÓN SUPERIOR
}

\author{
Francisco Javier Blanco Encomienda \\ María José Latorre Medina \\ Universidad de Granada
}

\begin{abstract}
RESUMEN: El actual proceso de convergencia con Europa en la creación de un espacio educativo compartido hace que se consideren como acciones prioritarias el incremento de la calidad de la enseñanza universitaria y la mejora de la excelencia docente. Profundizar y clarificar cuáles son los elementos que coadyuvan a conseguirlo se convierte en un tema central de estudio. El artículo que presentamos pretende ser una clara contribución en esta dirección, encuadrándose dentro de un trabajo de investigación de mayor envergadura llevado a cabo por profesores de la Universidad de Granada y financiado por el Ministerio de Ciencia y Tecnología dentro de la Convocatoria del Plan Nacional de Investigación Científica, Desarrollo e Innovación Tecnológica 2004-2007, con el que se persigue construir un programa de formación on line para el profesorado universitario a partir del estudio de las expectativas y necesidades manifestadas por éstos ante el área europea. Este artículo centra su atención en la primera etapa de la investigación, encaminada a la confección del instrumento de recogida de datos, describiendo el proceso de elaboración y depuración del cuestionario preparado ad hoc conforme a criterios de validez y fiabilidad.
\end{abstract}

ABSTRACT: The present process of convergence with Europe in creating a shared educational space provokes that the quality of higher education and the improvement of teaching excellence were considered as priority actions. Deepening and clarifying the elements that help to achieve it has become a focus of study. The paper we present tries to be a clear contribution in this direction, framed in a larger research conducted by academics from University of Granada and funded by the Ministry of Science and Technology within the Call of National Plan for Scientific Research, Development and Technological Innovation 2004-2007, which aims to elaborate an online training program for university teachers from the study of expectations and needs expressed by them about the European area. This article focuses on the 
first stage of the research, aimed to build the data collection tool, describing the process of development and debugging of the ad hoc questionnaire according to criteria of validity and reliability.

PALABRAS CLAVE: Educación superior, formación del profesorado universitario, necesidades docentes, expectativas docentes, Convergencia Europea.

KEYWORDS: Higher education, university teachers training, teachers' needs, teachers' expectations, European Convergence.

\section{MARCO teÓRICO}

\subsection{La docencia universitaria y el nuevo modelo educativo de enseñanza superior}

Según Alegre (2005), una de las piezas clave sobre la que pivota la construcción del Espacio Europeo de Educación Superior (EEES) es la mejora de la calidad del sistema universitario, como lo prueba el hecho de que, desde el principio, la calidad sea una referencia en todos los textos y así se enfatizara en el comunicado de los Ministros de Educación europeos tras la reunión de Praga, en el año 2001. La consecución del objetivo de lograr que Europa se convierta en lugar de referencia en el ámbito de la formación universitaria para estudiantes de todos los países pasa necesariamente por una garantía de la calidad fundamentada en mecanismos y procesos de evaluación, certificación y acreditación.

En la actualidad, el debate abierto ante los retos que plantea el proyecto de convergencia europea en materia de educación superior y las nuevas exigencias de la sociedad del conocimiento comportan un nuevo escenario y rediseño en la práctica educativa de las universidades europeas (Reichert y Tauch, 2003). La universidad española ha iniciado así un interesante proceso de transformación dirigido a coordinar sus políticas educativas de enseñanza superior y crear el denominado EEES. La Universidad de Granada no es ajena a este interés y en los últimos años ha emprendido numerosas iniciativas, de distinto calado, destinadas a favorecer esta adaptación. Posiblemente la implantación, con carácter experimental, del sistema de créditos europeo sea una de las que más interés y movilización está provocando.

No cabe duda de que el actual proceso de armonización europea ha motivado -y continúa haciéndolo- la revisión y adaptación de las características de la enseñanza universitaria a un nuevo marco de trabajo docente: la formación basada en las competencias que, ante la nueva realidad de la convergencia europea en torno a la educación, títulos y perfiles profesionales, se convierte en el componente principal de la enseñanza universitaria (Rodríguez, 2008). De acuerdo con la autora, uno de los retos más importantes que han de afrontar las universidades en los próximos años es redefinir su oferta formativa tratando de dar una respuesta más ajustada a las nuevas demandas que tanto la sociedad como los individuos le reclaman. Consciente de ello, en su trabajo explicita un modelo de formación basado en las competencias y los cambios que dicho modelo implica para el profesorado y el alumnado.

En esta línea, González y Raposo (2006) y Agüera, Alfageme y Calderón (2006), tras analizar algunos de los documentos específicos elaborados sobre el EEES, tanto 
de carácter institucional como fruto de las investigaciones y recomendaciones de los expertos, matizan que este nuevo marco educativo conlleva una amplia revisión de las funciones y la organización de las instituciones universitarias, así como del papel del profesorado y del alumnado, de sus funciones y tareas, como veremos a continuación.

\subsection{El papel del estudiante universitario ante el EEES}

Considerar la enseñanza universitaria en la nueva perspectiva europea comporta asumir un nuevo enfoque educativo basado en el trabajo global del estudiante y no sólo en las horas de clase, como tradicionalmente se ha hecho, o dicho de otro modo, implica asumir el paso de un modelo centrado en la enseñanza de los docentes a uno más centrado en el alumno y su trabajo, como aprendiz activo y autónomo (Morales, 2005; Smith, 2004; Torre y Gil, 2004), donde éste ha de ejercer una mayor iniciativa, una 'autonomía supervisada'. Tal como sostiene Madrid (2004), ha de poseer una actitud más emprendedora y una mayor cota de responsabilidad de su propio aprendizaje. Autores como Rico y Rico (2003) se refieren a esta modalidad de aprendizaje como aquélla "en la que el alumno se responsabiliza de la organización de su trabajo, de la adquisición de conocimientos y los asimila a su propio ritmo" (15).

En este nuevo escenario, los profesores tendrán que poner el énfasis en la participación y en el compromiso activo y crítico del estudiante con su aprendizaje (Alcalde y Armada, 2006), puesto que la calidad de la enseñanza universitaria se valorará a partir de la efectividad del aprendizaje del alumnado (López, 2005; Mohanan, 2003). Sabemos que asumir el papel protagonista por parte del estudiante en el proceso de enseñanza-aprendizaje es una tarea difícil, pero imprescindible en aras a elevar la calidad del aprendizaje universitario (Bigg, 2005).

\subsection{Reorientación en la labor docente del profesorado universitario}

La adecuación de las universidades españolas a las nuevas directrices marcadas desde Europa está propiciando una serie de reformas radicales a las que no están ajenos los profesores. Siguiendo a Zabalza (2007), el nuevo enfoque en la formación necesita invariablemente un cambio en el rol del profesorado que no va a ser fácil y tiene muchas implicaciones. Concretamente, a nivel de docencia, los cambios están siendo evidentes, girando éstos alrededor de unos propósitos básicos como son (León, Rodríguez y Romero, 2006):

a) Priorización de estrategias y metodologías didácticas participativas que promuevan un aprendizaje autónomo y la reconstrucción de los conocimientos por parte del alumnado.

b) Incorporación de la planificación basada en competencias que promueva la formación integral y la práctica de los alumnos. De acuerdo con Benítez, García y Fernández (2006), "el nuevo proceso de formación propuesto para el EEES requiere algunos cambios en la configuración de los planes de estudios universitarios. Dichos planes deben adecuarse a un nuevo proceso de enseñanza-aprendizaje centrado en el alumnado y su trabajo, en el que se 
desarrollen y se maticen las competencias y capacidades generales y específicas de cada una de las titulaciones. Las competencias y capacidades profesionales se convierten en un pilar básico de la educación superior" (209).

c) Cambios sustanciales en los procesos de aprendizaje, tanto si se trata de procesos de aprendizaje de destrezas y habilidades prácticas, que suelen adquirirse en el desarrollo de las propias actividades productivas mediante procesos de aprendizaje por la acción, como si se trata de procesos de formación de aptitudes que se adquieren a través de procesos formativos en los sistemas formales de enseñanza.

d) Necesidad de asumir los retos del aprendizaje a lo largo de toda la vida y de la utilización de las tecnologías de la información y la comunicación.

e) Introducción del crédito europeo como unidad del haber universitario. El sistema de créditos ECTS significa fundamentalmente una nueva concepción de la organización del tiempo, tanto de enseñanza como de aprendizaje (Agüera, Alfageme y Calderón, 2006).

El papel docente del profesor universitario se ha visto trastocado ante la llegada inminente del nuevo enfoque pedagógico. Madrid (2004) precisa en su trabajo que el profesor adquiere ahora un papel de acompañante, de supervisor y guía del aprendizaje del alumno, para alcanzar objetivos y competencias previamente definidas. Sin dejar de ser transmisor de conocimiento, su papel se dirige ahora a mayores niveles de tutoría, de apoyo y motivación para adquirir el conocimiento y habilidades necesarias; a la comprensión y capacidad para aplicar ese conocimiento; a la selección crítica de materiales y fuentes, a la organización de situaciones de aprendizaje, entre otras tareas.

Llegados a este punto, cabe mencionar que, además, el nuevo enfoque educativo comporta para el profesor universitario nuevas áreas de competencias profesionales (Tigelaar, Dolmans, Wolfhagen y Van der Vleuten, 2004; Zabalza, 2006). Alcalde y Armada (2006) afirman que el docente tiene que estar preparado para cambiar su manera de organizar y planificar sus clases presenciales y las sesiones no presenciales, de gestionar el trabajo y el estudio del alumno, de promover tareas colaborativas, de guiar al estudiante hacia un aprendizaje significativo y de fomentar, entre otros aspectos, el aprendizaje a lo largo de toda la vida. Por su parte, Morales (2005) apunta que son tres las áreas de competencias que el docente debe trabajar: una, centrada en el diseño de las tareas de estudio y de aprendizaje autónomo; otra, referida a la evaluación académica, entendida ésta como evaluación formativa; y una tercera, relacionada con la dimensión afectiva del aprendizaje, lo que se ha dado en Ilamar competencias relacionales como son la motivación, el gusto por la asignatura, la ansiedad, la relación profesor-alumno, etc.

Por todo ello, hoy en día es difícil encontrar una universidad que no haya diseñado acciones encaminadas a mejorar la competencia profesional de sus profesores, por ser una garantía para elevar la calidad de los procesos formativos que reciben los estudiantes, además de un requisito imprescindible para hacer factible la 
creación del EEES (Valcárcel, 2003). En consecuencia, describir los roles que deben caracterizar al profesor universitario demandado (Mohanan, 2003), las dimensiones del currículum que se ven afectadas por el nuevo papel docente que debe asumir el profesor de universidad (Mérida, 2006) y los indicadores de buenas prácticas de docencia universitaria bajo criterios ECTS (Gil, Bernaras, Elizalde y Garaigordobil, 2006) se convierten, entre otros, en temas centrales de investigación, reflexión y análisis para instituciones y profesionales.

\section{Objetivos de La inVESTIGACIÓN}

El trabajo que presentamos forma parte de una investigación de mayor envergadura llevada a cabo por un grupo de profesores de la Universidad de Granada y financiado por el Ministerio de Ciencia y Tecnología dentro de la Convocatoria del Plan Nacional de Investigación Científica, Desarrollo e Innovación Tecnológica 2004-2007 (Ref. SEJ2004-08035-C02-02), con la que se pretende construir un programa de formación on line para los profesores universitarios a partir del estudio de las expectativas y necesidades manifestadas por éstos ante el proceso de convergencia como medida para incrementar la efectividad de los programas formativos en la educación superior y la calidad de la enseñanza universitaria. Este propósito general se concreta en los siguientes objetivos de investigación:

1. Estudiar las expectativas de los profesores universitarios en distintos centros universitarios y áreas de conocimiento ante la convergencia europea en materia de educación superior.

2. Conocer las necesidades que derivan de esta convergencia en función de las diferentes situaciones administrativas y profesionales (categorías profesionales) y los distintos campos profesionales.

3. Establecer, a partir de la información proporcionada por los propios docentes, un sistema de competencias que permitan orientar la formulación de propuestas formativas del profesorado en la enseñanza superior.

4. Construir un programa de formación on line que, basado en las necesidades sentidas por los profesores universitarios y en sus expectativas, impulse su desarrollo profesional y les permita responder con profesionalidad al reto que representa la integración del sistema universitario español en el espacio europeo de enseñanza superior.

\section{Metodología}

La investigación se estructura en torno a dos grandes etapas. La primera de ellas encaminada a la construcción de un instrumento (cuestionario) que nos permita recabar información sobre las necesidades y expectativas del profesorado que participa en la experiencia piloto de implantación del crédito europeo en la Universidad de Granada. La segunda, destinada al diseño de un programa de formación sustentado en las necesidades previamente manifestadas por los docentes.

El proceso seguido en la investigación ha sido el siguiente: 


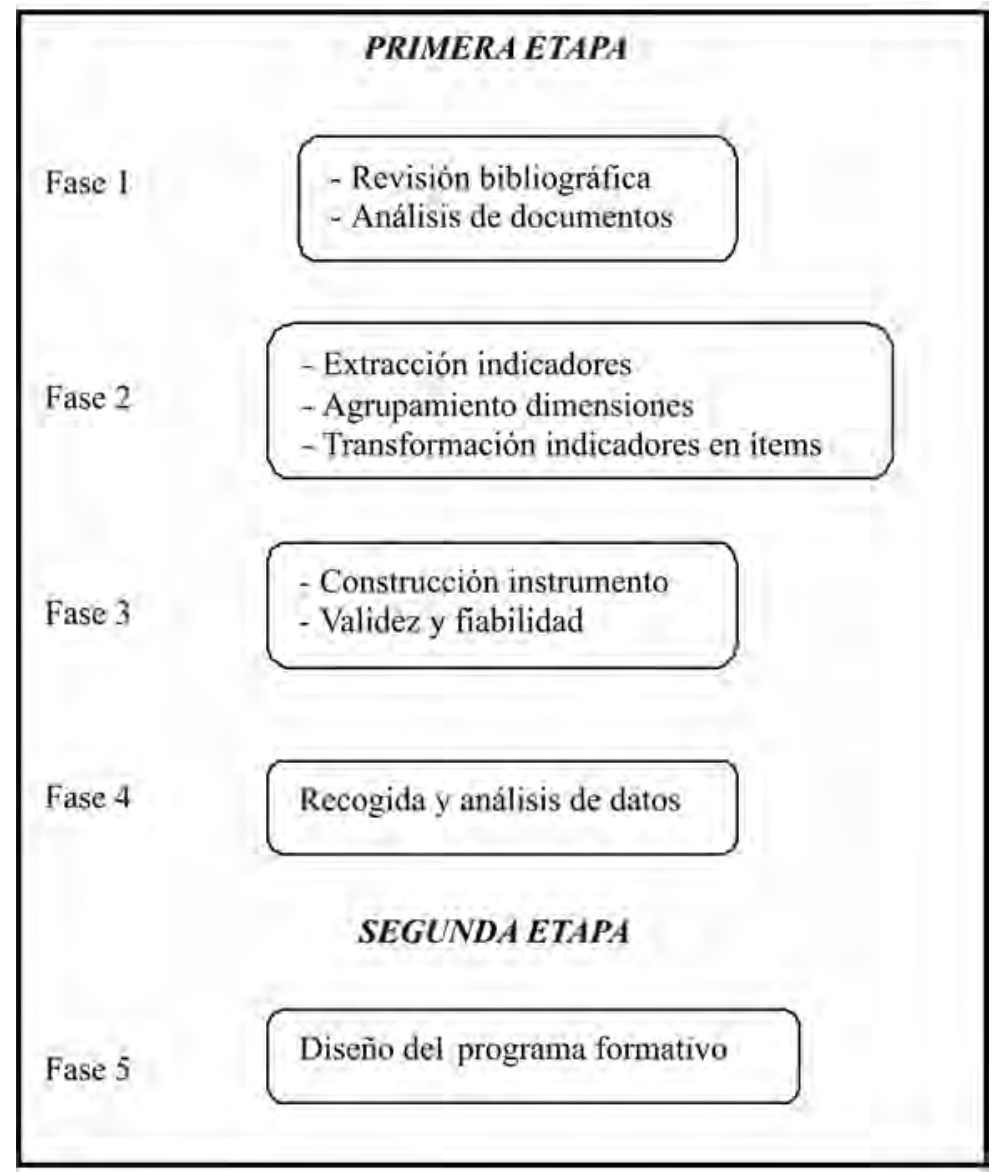

Figura 1. Proceso seguido en la investigación.

En el presente artículo nos centramos en la primera de estas etapas, dirigida a satisfacer los objetivos uno y dos. En concreto, informamos del proceso de elaboración y depuración del cuestionario confeccionado ad hoc.

\subsection{Proceso seguido para la recogida y análisis de información}

La primera fase de construcción del cuestionario se inicia con un proceso de revisión bibliográfica sobre distintas aportaciones, experiencias y proyectos vinculados al proceso de convergencia y a la implantación del nuevo sistema de créditos ECTS. Este tarea transcurre de manera paralela a una segunda focalizada en el análisis de tres tipos de documentos: informes de evaluación emitidos desde las titulaciones de la Universidad de Granada que participan en la experiencia piloto de implantación del crédito europeo, transcripciones de algunas de las sesiones de evaluación llevadas a cabo por los profesores que participan en dicha experiencia y documentos técnicos (Goetz y LeCompte, 1988). Estos documentos técnicos son instrumentos diseñados 
para orientar la reflexión y recabar información sobre las necesidades y expectativas manifestadas por los docentes a través de los coordinadores de la experiencia piloto en cada una de las trece titulaciones que vienen participando en la implantación del crédito europeo desde el curso 2004-2005 en la Universidad de Granada, que han sido: Ciencias Ambientales, Enfermería, Maestro Audición y Lenguaje (Facultad de Educación y Humanidades Ceuta), Maestro Educación Especial (Facultad de Educación y Humanidades Ceuta), Maestro Audición y Lenguaje (Facultad de Educación y Humanidades Melilla), Maestro Educación Infantil (Facultad de Educación y Humanidades Melilla), Maestro Educación Infantil (Facultad de Ciencias de la Educación de Granada), Maestro Educación Primaria (Facultad de Ciencias de la Educación de Granada), Maestro Lengua Extranjera (Facultad de Ciencias de la Educación de Granada), Medicina, Psicología, Química y Biología.

Estos documentos técnicos, dirigidos a los coordinadores, se sistematizaron en torno a seis cuestiones abiertas:

a) Necesidades y expectativas relacionadas con las disposiciones ofrecidas hasta el momento por la política educativa sobre el espacio europeo de educación superior.

b) Necesidades y expectativas generadas a nivel institucional (Universidad, Facultad, Departamento).

c) Necesidades y expectativas vinculadas con el diseño, desarrollo o evaluación curricular.

d) Necesidades y expectativas relacionadas con el desarrollo profesional (formación, promoción, compromisos, dedicación, implicación, perfeccionamiento, autoevaluación, evaluación, percepciones...).

e) Necesidades y expectativas a nivel personal de los docentes universitarios ante la convergencia europea (satisfacción/insatisfacción, resistencias, seguri$\mathrm{dad} /$ inseguridad, dificultades, etc.).

f) Otras aportaciones.

Esta doble labor recopilatoria de información, resultante de la revisión bibliográfica y del análisis de los documentos mencionados, será el punto de partida para extraer un conjunto de indicadores que servirán de referencia a la elaboración de los ítems que integrarán el cuestionario. Estos indicadores son inicialmente agrupados en cinco dimensiones (macroestructural, microestructural, curricular, profesional y personal) y posteriormente reducidos a cuatro.

La primera de estas cuatro dimensiones, dimensión estructural, incluye aquellas cuestiones relacionadas con las condiciones, decisiones y actuaciones emprendidas por altas instancias y relacionadas con el proceso de convergencia y la implantación del crédito europeo. La segunda, dimensión curricular, pretende aglutinar todas las aportaciones que afectan al diseño, desarrollo o evaluación curricular (objetivos, contenidos, metodología...). El ámbito profesional engloba a las cuestiones relacionadas con la formación, dedicación, evaluación, promoción... de los profesores. Y, finalmente, la dimensión personal abarca todo lo relativo al componente afectivo y emocional del cambio (grado de satisfacción/insatisfacción, rechazos, autopercepciones, dificultades personales, resistencias, etc.). 
Una vez efectuada la correspondiente extracción de indicadores y su adjudicación a las distintas dimensiones generadas, se inicia el proceso de conversión de estos indicadores en ítems, del cual resulta una primera versión del cuestionario. Esta primera versión ha sido objeto de una amplia y profunda revisión por parte de los miembros del equipo de investigación, quienes lo han depurado en cuanto a su formulación.

\subsection{Estudio de la validez del instrumento}

La versión resultante del cuestionario diseñado ha sido validada mediante la técnica Delphi (Linstone y Turoff, 1977) y el sistema de jueces expertos en la materia (Beltrán y Rodríguez, 2004). En concreto, se ha sometido a revisión de jueces entre un grupo de diez expertos en distintos campos profesionales (Ciencias Experimentales, Medicina, Educación, Derecho, Sociología, Psicología y Filología) que, en revisiones sucesivas, permitieron reformular enunciados y afinar el instrumento hasta construir la versión definitiva.

\subsection{Cálculo de la fiabilidad del instrumento}

Para determinar la fiabilidad del cuestionario se ha recurrido a dos métodos: " $\alpha$ de Cronbach" y "dos mitades". El coeficiente $\alpha$ general fue de 0,910 para los 82 elementos del cuestionario. Además, se calculó el mismo coeficiente para cada uno de los 82 ítems si cada uno de ellos se elimina. Las puntuaciones obtenidas siempre fueron valores iguales o superiores a 0,905 (llegando a alcanzar el valor 0,914), tanto en las referidas a los ítems cuanto las relativas al coeficiente general, por lo que podemos afirmar que el cuestionario tiene un elevado grado de consistencia interna, ya que un rango de 0,600 se considera aceptable (Thorndike, 1997).

Aplicamos, además, el método de las dos mitades (fórmula de predicción de Spearman-Brown), pues los métodos de partición propenden a una estimación rebajada de la fiabilidad, dado que la fiabilidad del instrumento está relacionada con su extensión (Bisquerra, 1987; Fox, 1987). Los resultados incluidos en la tabla siguiente avalan igualmente la fiabilidad del instrumento:

Cuadro 1. Fiabilidad del cuestionario y coeficiente de consistencia interna para cada mitad.

\begin{tabular}{|l|c|c|c|}
\hline \multirow{2}{*}{ Alfa de Cronbach } & \multirow{2}{*}{ Parte 1 } & Valor & 0,917 \\
\cline { 3 - 4 } & & N de elementos & $41 \mathrm{a}$ \\
\cline { 3 - 4 } & \multirow{2}{*}{ Parte 2 } & Valor & 0,743 \\
\cline { 3 - 4 } & N de elementos & $41 \mathrm{~b}$ \\
\hline \multirow{2}{*}{ Correlación entre formas } & N total de elementos & 82 \\
\hline Coeficiente de Spearman-Brown & & 0,633 \\
\cline { 2 - 4 } & \multicolumn{2}{|c}{ Longitud igual } & 0,775 \\
\hline Dos mitades de Guttman & \multicolumn{2}{|c|}{ Longitud desigual } & 0,775 \\
\hline
\end{tabular}




\subsection{Descripción del instrumento de recogida de datos}

El cuestionario final, titulado "Cuestionario sobre necesidades y expectativas de los docentes universitarios derivadas del proceso de convergencia con Europa", está compuesto por 82 ítems, 48 relativos a necesidades y 34 referidos a expectativas, con formato de escala tipo Lickert, con cinco opciones de respuesta $(1=$ Totalmente en desacuerdo, 2= En desacuerdo, 3= Ni de acuerdo ni en desacuerdo, 4= De acuerdo y $5=$ Totalmente de acuerdo). Más concretamente, los 82 ítems que conforman el cuestionario quedan distribuidos en seis apartados diferentes, que son:

a) Necesidades estructurales (ítems 1 al 11). Son ítems ilustrativos de esta dimensión:

- Ítem 2: La incorporación de la Universidad Española al Espacio Europeo de Educación Superior (EEES) exige aumentar la plantilla del personal docente.

- Ítem 6: La incorporación del ECTS al sistema universitario español requiere una reducción del número de alumnos por aula.

- Ítem 7: Es importante que la universidad diseñe un sistema de incentivos que contemple la implicación de los docentes en procesos de innovación y mejora de su docencia.

b) Necesidades curriculares (ítems 12 al 28). Algunos de los ítems que conforman este segundo apartado son:

- Ítem 14: La adopción del ECTS supone primar planteamientos educativos dirigidos a favorecer la formación para el mercado laboral frente a un enfoque más humanístico.

- Ítem 16: Para la adopción del nuevo modelo educativo es necesario que los profesores revaloricen el trabajo práctico de los estudiantes.

- Ítem 23: Con la adopción del crédito europeo se hace necesario diversificar los instrumentos y técnicas de evaluación (seminarios, carpetas de aprendizaje, autoevaluación, mapas conceptuales...).

c) Necesidades profesionales/personales (ítems 29 al 48). Este grupo de declaraciones está integrado por ítems como los siguientes:

- Ítem 34: El profesorado necesita que se pondere de forma adecuada las diferentes tareas docentes que realiza (planificación, orientación, seguimiento, evaluación...) dentro de su carga lectiva y que éstas tengan un reconocimiento en su promoción profesional.

- Ítem 35: Es necesario crear un cuerpo de profesionales universitarios competentes que formen y asesoren al profesorado a nivel didáctico para mejorar la calidad de la docencia universitaria.

- Ítem 44: La estabilidad del profesorado es imprescindible si se desea incrementar su nivel de compromiso con el nuevo modelo educativo.

d) Expectativas estructurales (ítems 49 al 58). La cuarta dimensión incluye ítems como los que se muestran a continuación:

- Ítem 52: Espero que la creación de EEES permita una mayor movilidad de nuestros estudiantes, titulados y profesorado. 
- Ítem 55: Estimo que las condiciones estructurales y de apoyo desplegadas hasta el momento (realidad de las aulas, acceso a las TICs...) son insuficientes para responder a las exigencias del crédito europeo.

- Ítem 57: Desconfío de que las iniciativas de información y formación para la implantación del crédito europeo incrementen la implicación de los docentes.

e) Expectativas curriculares (ítems 59 al 69). Ejemplos de ítems que conforman este apartado son:

- Ítem 63: Creo que será difícil para el profesorado adaptar sus materias a la forma de trabajar que propone el futuro modelo educativo.

- Ítem 65: Desconfío de que los alumnos sepan trabajar de forma más autónoma y asuman las exigencias que conlleva el nuevo modelo educativo.

- Ítem 69: Considero que la planificación colaborativa entre colegas resulta difícil de conseguir en la actual cultura profesional universitaria.

f) Expectativas profesionales/personales (ítems 70 al 82). Esta última dimensión aglutina ítems como los siguientes:

- Ítem 71: Confío en que el tiempo invertido en la mejora de la labor docente sea valorado en la misma medida que la actividad investigadora.

- Ítem 76: Considero que el incremento de tiempo que debe invertirse en las tareas docentes con el ECTS repercute negativamente en la cantidad y calidad de la labor investigadora.

- Ítem 81: Creo que la multiplicación de tareas y compromisos de los docentes hacen difícil su implicación en los procesos de cambio que exige el proceso de convergencia.

El cuestionario cuenta con un bloque inicial de información identificativa de los docentes a los que va dirigido. En este apartado, se solicita información acerca del sexo, edad, titulación máxima que poseen, número de años de experiencia docente en la Universidad, campo de docencia universitaria, facultad/escuela a la que pertenecen, categoría profesional, dedicación y curso académico desde el que están participando en la experiencia piloto para la implantación del crédito europeo, a continuación de lo cual se presenta la escala de valoración.

Finalmente, señalar que la redacción de los ítems está orientada hacia la consecución de información que pueda ser tratada mediante las pruebas estadísticas pertinentes, tanto para distribuciones normales como para las no normales. Esto implica que la redacción de los ítems que conforman cada dimensión puede ser valorada con una puntuación que signifique, respecto a la afirmación del ítem, lo mismo en cada una de ellas.

\subsection{Aplicación del cuestionario}

A partir de este momento, se procedió al envío del cuestionario a los 619 profesores universitarios que imparten docencia en las veintiuna titulaciones que desde el curso 2004-2005 vienen participando en la implantación del crédito europeo en la Universidad de Granada. 
Esta iniciativa permitió recopilar un total de 200 cuestionarios, remitidos por el propio profesorado de los distintos centros implicados (cuadro 2) y que quedaron finalmente agrupados en cuatro campos de conocimiento: Ciencias de la Salud, Ciencias Sociales y Jurídicas, Ciencias Experimentales/Técnicas y Humanidades.

Cuadro 2. Centros participantes en la investigación.

\author{
Facultad de Ciencias \\ Facultad de Medicina \\ Facultad de Ciencias de la Educación \\ Facultad de Psicología \\ Escuela Universitaria de Ciencias de la Salud \\ Facultad de Derecho \\ Facultad de Biblioteconomía y Documentación \\ Facultad de Filosofía y Letras \\ Facultad de Educación y Humanidades de Ceuta \\ Facultad de Educación y Humanidades de Melilla
}

\title{
3.6. Análisis de los datos
}

Los datos recabados mediante el cuestionario fueron vaciados y posteriormente sometidos a tratamiento cuantitativo a través del paquete SPSS. Los análisis estadísticos efectuados han sido los siguientes: descriptivos, contingencias, clusters de sujetos y factoriales. Y los resultados arrojados han servido de punto de partida para la segunda etapa de la investigación: el diseño de una propuesta formativa on line para el profesorado universitario, hoy en día finalizado.

A este respecto, y centrándonos en el aspecto metodológico del programa, cabe mencionar brevemente que, fieles a los actuales parámetros que definen la formación del profesorado, han sido diseñados distintos módulos formativos dirigidos a incrementar el desarrollo competencial del docente universitario en cuatro aspectos: exposición magistral, fomento del trabajo autónomo (elaboración de guías de trabajo y optimización de las técnicas de aprendizaje), organización del trabajo en grupo (discusión grupal, seminarios, prácticas en laboratorios y trabajo de campo) y tutoría. Cada uno de estos ocho módulos mantienen una misma estructura interna en la que se lleva a cabo una aproximación conceptual al sentido y finalidad de la competencia, se indican claves para ejecutarla, se ejemplifica a través de un caso, se proporcionan pautas para trasladar a escenarios particulares, se indican algunas fuentes para profundizar y se elaboran instrumentos para facilitar la evaluación de la competencia en cuestión. 


\section{Conclusiones}

El propósito principal de nuestra investigación fue construir un programa de formación on line para el profesorado universitario a partir del estudio de las expectativas y necesidades manifestadas por éstos ante el espacio europeo de educación superior. Este objetivo general se dividió en varios objetivos específicos, siendo necesario para la consecución de los dos primeros, la construcción de un instrumento que permitiera recabar información valiosa sobre las necesidades y expectativas del profesorado que participa en la experiencia piloto de implantación del crédito europeo en la Universidad de Granada.

El presente trabajo ha centrado su atención en ello, describiendo el proceso de elaboración y depuración del cuestionario confeccionado ad hoc conforme a criterios de validez y fiabilidad. Las evidencias obtenidas permiten constatar que el cuestionario es un instrumento útil para los objetivos de la investigación.

Más concretamente, de este trabajo se pueden extraer las siguientes conclusiones:

- Respecto a la validez, el cuestionario ha sido validado mediante la técnica Delphi y por el sistema de jueces expertos en la materia hasta alcanzar finalmente una validez aceptable.

- En cuanto a la fiabilidad, los dos procedimientos utilizados han mostrado unos resultados muy buenos. En concreto, cabe destacar que el cuestionario:

- Ha obtenido un coeficiente de consistencia interna muy elevado.

- Presenta una alta fiabilidad medida por el procedimiento "dos mitades".

Haciendo un balance general del trabajo realizado, podemos afirmar que el camino recorrido nos ha permitido manejar información valiosa sobre el proceso de convergencia y elementos clave del proceso de implantación del crédito ECTS, disponer de un instrumento con el que obtener datos sobre las necesidades y expectativas del profesorado universitario ante la convergencia europea, avanzar en el conocimiento sobre la naturaleza de la formación de los profesores en la educación superior y elaborar, con la intención de allanar el camino, una propuesta de formación sustentada en las necesidades y expectativas manifestadas por los propios docentes que participan en la experiencia piloto del crédito europeo, pues "como era de esperar -advierte Rodríguez (2008) - no todo son vítores en este cambio de enfoque. Este proceso no está al margen de las dificultades que se presuponen a todo tipo de cambios. Es algo con lo que hay que contar ya que no es lo mismo acomodarse a un cambio técnico que a un cambio de mentalidad o de actitud" (141). Todo un reto educativo.

\section{REFERENCIAS BIBLIOGRÁFICAS}

AGÜERA, E.; ALFAGEME, B. y CALDERÓN, D. (2006). El marco europeo impulsor de creatividad e innovaciones en la educación. Actas del VI Congreso Internacional Virtual de Educación, CIVE 2006, en http://www.cibereduca.com (04/10/2008). 
ALCALDE, A. y ARMADA, R. (2006). Elaboración de una escala de evaluación de la competencia docente universitaria a la luz del espacio europeo de educación superior. En Actas del IX Congreso Interuniversitario de Organización de Instituciones Educativas. CD-Rom. Oviedo: Universidad de Oviedo.

ALEGRE, O. M. (2005). Construir un espacio europeo de Educación Superior. En L. M. Villar, P. S. de Vicente y O. M. Alegre (Dirs.), Conocimientos, capacidades y destrezas estudiantiles (267-315). Madrid: Pirámide.

BELTRÁN, R. y RODRÍGUEZ, J. L. (2004). Validez. En F. Salvador, J. L. Rodríguez y A. Bolívar (Dirs.), Diccionario Enciclopédico de Didáctica. Volumen II (665665). Málaga: Aljibe.

BENÍTEZ, J. L.; GARCÍA, A. B. y FERNÁNDEZ, M. (2006). Análisis de la percepción y conocimientos del profesorado sobre el bullying para mejorar su competencia. En J. L. Benítez, A. B. García, F. Justicia y J. de la Fuente (Coords.), La Universidad ante el reto del Espacio Europeo de Educación Superior: Investigaciones recientes (209-229). Madrid: Eos.

BIGG, J. B. (2005). Calidad del aprendizaje universitario. Madrid: Narcea.

BISQUERRA, R. (1987). Introducción a la estadística aplicada a la investigación educativa. Un enfoque informático con los paquetes BMDP y SPSS. Barcelona: PPU.

FOX, D. J. (1987). El proceso de investigación. Pamplona: Ediciones Universidad de Navarra.

GIL, P.; BERNARAS, E.; ELIZALDE, L. M. y GARAIGORDOBIL, M. (2006). Buenas prácticas de docencia universitaria: opinión del alumnado sobre las actividades de enseñanza-aprendizaje y sobre el desempeño docente. Actas del IV Congreso Internacional de Docencia Universitaria e Innovación. "La competencia docente". CD-Rom. Barcelona: Facultad de Ciencias Económicas y Empresariales de la Universidad de Barcelona.

GOETZ, J. P. y LECOMPTE, M. D. (1988). Etnografía y diseño cualitativo en investigación educativa. Madrid: Morata.

GONZÁLEZ, M. y RAPOSO, M. (2006). Los retos de la convergencia europea: valoraciones de los responsables académicos universitarios. Actas del IX Congreso Interuniversitario de Organización de Instituciones Educativas. CD-Rom. Oviedo: Universidad de Oviedo.

LEÓN, M. J.; RODRÍGUEZ, M. J. y ROMERO, M. A. (2006). Los mapas conceptuales como instrumento de evaluación y de aprendizaje en la universidad, dentro del nuevo espacio europeo de educación superior. Actas del IX Congreso Interuniversitario de Organización de Instituciones Educativas. CD-Rom. Oviedo: Universidad de Oviedo.

LINSTONE, H. A. y TUROFF, M. (1977). The Delphi method. Techniques and applications. Reading, Massachusetts: Addison-Wesley.

LÓPEZ, F. (2005). Metodología participativa en la Enseñanza Universitaria. Madrid: Narcea.

MADRID, D. (2004). La formación inicial del profesorado de Lengua Extranjera. Profesorado. Revista de currículum y formación del profesorado, 8 (1), 1-19, en http://www.ugr.es/ recfpro/rev81ART7.pdf (24/05/2009). 
MÉRIDA, R. (2006). Nueva percepción de la identidad profesional del docente universitario ante la convergencia europea. Revista Electrónica de Investigación Educativa, 8 (1). Disponible en http://redie.uabc.mx/vol8no1/contenido-merida.html (07/09/2008).

MOHANAN, K. P. (2003). Assessing Quality of Teaching in Higher Education.

MORALES, P. (2005). Implicaciones para el profesorado de una enseñanza centrada en el alumno. Disponible en http://www.net.upcomillas.es/innovacioneducativa/documentos/enseñanza_centrada_\%20aprendizaje.pdf (26/10/2009).

REICHERT, S. y TAUCH, C. (2003). Bologna four years after: Steps toward sustainable reform of higher education in Europe. Trends 2003: Progress towards the European Higher Education Area. Ginebra: European University Association.

RICO, M. y RICO, C. (2003). El portafolio discente. Serie Docencia Universitaria. Madrid: Marfil.

RODRÍGUEZ, R. M. (2008). Un modelo de formación basado en las competencias: hacia un nuevo paradigma en la enseñanza universitaria. Contextos Educativos, $11,131-147$.

SMITH, B. (2004). Putting Student Learning at the Centre of Higher Education. Conference on Teaching and Learning in Higher Education. Disponible en http://www.cdtl.nus.edu.sg/tlhe/keynote1.htm (04/06/2007).

THORNDIKE, R. M. (1997). Measurement and evaluation in psychology and education. $6^{\text {a }}$ Edición. New York: Macmillan.

TIGELAAR, D.; DOLMANS, D.; WOLFHAGEN, I. y VAN DER VLEUTEN, C. (2004). The development and validation of a framework for teaching competencies in higher education. Higher Education, 48, 253-268.

TORRE, J. C. y GIL, E. (Eds.) (2004). Hacia una enseñanza universitaria centrada en el aprendizaje. Madrid: Universidad Pontificia Comillas.

VALCÁRCEL, A. (Coord.) (2003). La preparación del profesorado universitario español para la convergencia europea en educación superior. Memoria de investigación. Córdoba: Universidad de Córdoba.

ZABALZA, M. A. (2006). Competencias docentes del profesorado universitario. Calidad y desarrollo profesional. 2a Edición. Madrid: Narcea.

ZABALZA, M. A. (2007). Enseñanza universitaria. El escenario y sus protagonistas. Madrid: Narcea. 